\title{
Systemic Cyclosporine Therapy for Scleritis: A Proposal of a Novel System to Assess the Activity of Scleritis
}

\author{
Haruka Aoki Miki Hiraoka Masato Hashimoto Hiroshi Ohguro \\ Department of Ophthalmology, School of Medicine, Sapporo Medical University, Sapporo, \\ Japan
}

\section{Key Words}

Unilateral scleritis $\cdot$ Systemic cyclosporine administration $\cdot$ Scoring system

\begin{abstract}
In the present study, two female patients with unilateral scleritis without systemic complications were examined. Both patients were suffering from ocular pain and received corticosteroid therapy. The first patient, a 45-year-old woman, was diagnosed with scleritis and iritis in her right eye. Topical corticosteroid treatment could eradicate the iritis but not the scleritis. Oral corticosteroid administration and corticosteroid pulse therapy were applied with little effect. The application of systemic cyclosporine had a satisfactory effect in controlling the scleritis. The other patient, a 60-year-old woman, was suffering from scleritis and lid swelling in her right eye. Not only did topical and systemic corticosteroid therapy prove insufficient, they also resulted in the elevation of her intraocular pressure. After termination of corticosteroid therapy, the systemic cyclosporine was applied orally. Subsequently, the patient's scleritis improved without any severe side effects. Scleritis is a painful and destructive inflammatory disease of the sclera that causes congestion of the scleral venous plexus. In this study, we have established a new grading system for assessing activity in scleritis that can score the extent of ocular pain and the area of congestion. This system provides a practical method for following the clinical course and monitoring the outcome of therapy. We report two cases of unilateral scleritis that were resistant to corticosteroid therapy but responsive to systemic administration of cyclosporine. Findings from these cases indicate that cyclosporine is an effective drug for controlling severe scleritis.

(c) 2015 S. Karger AG, Basel
\end{abstract}

KARGER 125:s $\quad \begin{aligned} & \text { Miki Hiraoka, MD, PhD } \\ & \text { Department of Ophthalmology, School of Medicine } \\ & \text { Sapporo Medical University, S1 W16 Chuo-ku } \\ & \text { Sapporo, Hokkaido 060-8543 (Japan) } \\ & \text { E-Mail mikihira@sapmed.ac.jp }\end{aligned}$


Aoki et al.: Systemic Cyclosporine Therapy for Scleritis: A Proposal of a Novel System to Assess the Activity of Scleritis

\section{Introduction}

Scleritis is a serious inflammatory disease that can cause severe ocular pain and occasionally impaired vision. The etiology of scleritis can be idiopathic, autoimmune (it is often associated with systemic diseases such as rheumatoid arthritis, granulomatosis with polyangiitis, and systemic lupus erythematosus), or infectious [1]. The disease can be classified into anterior and posterior types according to the anatomical location of the lesions [2]. Anterior scleritis commonly presents with severe pain not restricted to the eye but also associated with headaches and periorbital pain. Furthermore, anterior scleritis causes congestion of the blood vessels and redness and edema of the sclera. The affected lesion can be sectoral or consist of the entire external anterior sclera. In some cases, scleral thinning and necrosis emerge. The condition of anterior scleritis can be evaluated through the redness of the lesion and ocular pain.

Corticosteroids in topical and/or systemic administration are widely accepted as an effective treatment for noninfectious scleritis $[3,4]$. However, there are several cases in which corticosteroid treatment has a minimal effect or its administration must even be terminated due to accompanying side effects. Recently, other immunosuppressive agents have been shown to be effective in improving the outcome of severe scleritis. There are several reports about the successful use of cyclosporine in treating various ocular inflammatory diseases [5].

Assessment of the degree of activity in scleritis is important for patient care. A quantitative grading system of the severity of scleritis is beneficial not only to describe the extent of inflammation in individual cases, but also to trace the therapeutic value.

In the present report, we describe two cases of idiopathic unilateral anterior scleritis that were unresponsive to topical or systemic corticosteroid therapy but responsive to systemic cyclosporine therapy. Their clinical course was monitored using a unique grading system we designed to assess the manifestation of scleritis.

\section{Materials and Methods}

The medical records of two patients with unilateral anterior scleritis were retrospectively reviewed. Scleritis symptoms were graded with scores as described in table 1.

\section{Results}

Case 1

A 45-year-old woman developed ocular pain and blurred vision in her right eye a month prior to presentation. She initially visited a separate clinic and received intravenous injections of antibiotics. These proved ineffectual, and she was referred to another hospital, where she was diagnosed with iritis with ciliary injection, keratic precipitate, hypopyon, and posterior synechia in her right eye. Topical betamethasone and oral prednisolone (30 $\mathrm{mg} /$ day) were applied. Although this treatment proved effective in healing the iritis, the redness and pain in her right eye deteriorated and she was referred to our hospital. Her left eye had mild visual impairment due to a childhood trauma. She did not have any medical history of systemic disease.

The initial ophthalmic examination disclosed a best-corrected visual acuity (BCVA) of $20 / 20$ in the right eye and 20/32 in the left eye. The intraocular pressure was $15 \mathrm{~mm} \mathrm{Hg}$ in the right eye and $18 \mathrm{~mm} \mathrm{Hg}$ in the left eye. A slit-lamp examination demonstrated no sign of 
Aoki et al.: Systemic Cyclosporine Therapy for Scleritis: A Proposal of a Novel System to Assess the Activity of Scleritis

uveitis or retinal disease besides conjunctival injection and scleral venous plexus congestion in the right eye. Both eyes showed substantial anterior chamber depth without peripheral anterior synechia. The clinical course is described in figure 1 . Results of the laboratory investigations of serum including antinuclear antibodies, anti-double-stranded DNA antibodies, rheumatoid factors, and antineutrophil cytoplasmic antibodies were unremarkable. Serological analysis indicated that there was no active infection.

The patient continued to receive topical betamethasone and oral prednisolone (10 $\mathrm{mg} /$ day), and subconjunctival triamcinolone injections were additionally administered, resulting in modest improvement. Neither topical cyclosporine nor topical antibiotics showed any effect on her ocular symptoms. One month later, brain magnetic resonance imaging indicated that there was no thickening of the posterior sclera or other abnormalities. When oral prednisolone was tapered, the patient's scleral congestion and ocular pain were aggravated. After admission, two sets of pulse therapy with intravenous methylprednisolone $(1 \mathrm{~g} /$ day for 3 days) following oral prednisolone $(40 \mathrm{mg} /$ day) were applied, yielding a moderately positive response. She then received systemic cyclosporine $(3 \mathrm{mg} / \mathrm{kg} /$ day), administered orally to treat aggravated scleritis, and her ocular symptoms subsequently declined. When the oral prednisolone treatment was terminated and the cyclosporine dosage was reduced to $2 \mathrm{mg} / \mathrm{kg} /$ day, the scleritis relapsed. When cyclosporine was increased to $3 \mathrm{mg} / \mathrm{kg} / \mathrm{day}$, the trough value was $37 \mathrm{ng} / \mathrm{ml}$, and her right eye developed peripheral cornea edema (fig. 2a). Upon increasing the amount of cyclosporine to $4 \mathrm{mg} / \mathrm{kg} / \mathrm{day}$, her ocular symptoms improved (fig. 2b). Although there was another relapse 3 months later, the cyclosporine dosage was retained. Since then, no relapse has occurred even after tapering the cyclosporine. During the clinical course, there was a temporal intraocular pressure elevation which could be maintained by topical antiglaucoma medication. Blood tests were examined monthly to check the trough value of cyclosporine and to monitor the cell counts and biochemical markers, particularly the renal function marker. No side effects resulting from systemic cyclosporine administration were observed.

\section{Case 2}

A 60-year-old woman presented with redness in her right eye. Before admission to our hospital, topical betamethasone was initially administered at another clinic, but there was no response. Moreover, 3 weeks later, a swelling appeared in the right upper eyelid and right lacrimal gland. After oral prednisolone (15 mg/day) was applied, the eyelid swelling diminished, but the redness and pain in the patient's right eye increased and she was referred to our hospital. She did not have any medical history of systemic disease or ocular disease.

The initial ophthalmic examination disclosed a BCVA of 20/20 in the right eye and $20 / 16$ in the left eye. The intraocular pressure was $21 \mathrm{~mm} \mathrm{Hg}$ in the right eye and $18 \mathrm{~mm} \mathrm{Hg}$ in the left eye. A slit-lamp examination indicated conjunctival injection and scleral venous plexus congestion in the right eye, but no sign of uveitis or retinal disease. The clinical course is described in figure 3. Results of the laboratory investigations of serum including antinuclear antibodies, rheumatoid factors, antineutrophil cytoplasmic antibodies, the level of IgG4, and SSA/Ro and SSB/La antibodies were unremarkable. Serological analysis showed no active infection. However, although a computed tomographic scan detected right lacrimal gland enlargement, symptoms such as dry eye or corneal erosion were not seen (fig. 2c). Additionally, the patient showed no symptoms of diplopia or impaired ocular movement.

Topical betamethasone drops and oral prednisolone $(30 \mathrm{mg} /$ day) were applied frequently and proved effective. Neither topical cyclosporine nor topical antibiotics showed any effect on her ocular symptoms. During oral prednisolone tapering, the intraocular pressure was elevated to $35 \mathrm{~mm} \mathrm{Hg}$ in the right eye and $25 \mathrm{~mm} \mathrm{Hg}$ in the left eye. The intraocular 
Aoki et al.: Systemic Cyclosporine Therapy for Scleritis: A Proposal of a Novel System to Assess the Activity of Scleritis

pressure elevation was controlled with topical antiglaucoma medication and the termination of oral and topical corticosteroids. Due to a scleritis relapse, topical betamethasone administration was resumed, but had little effect. Subsequently, systemic cyclosporine ( $3 \mathrm{mg} / \mathrm{kg} /$ day) was administered orally to treat the aggravated scleritis, and this proved effective. A blood test was administered monthly to check the trough value of the cyclosporine and to monitor the influence of the medication. The dosage of cyclosporine administration was reduced gradually without recurrence of scleritis or development of side effects.

\section{Discussion}

In the present study, we described two cases that shared similar phenotype traits. First, in both cases, the patients had unilateral anterior scleritis. Their intraocular inflammation was not controlled by the administration of either topical or systemic corticosteroids but showed a greater response to systemic cyclosporine administration. Neither was identified with any systemic disease that could be associated with scleritis.

The causes of scleritis vary and include infection, autoimmune diseases, and idiopathic diseases. The redness is mostly observed in anterior scleritis with severe pain. When inflammation is reduced, these symptoms will decline. To evaluate the severity and the effect of treatment, it has been necessary to establish an accurate and reproducible system for quantifying the severity of scleritis. At present, there are a few grading systems that make these evaluations [6, 7]. The scoring system proposed by Wakefield and McCluskey [6] is useful in order to assess the presence of complications with other ocular conditions such as uveitis, intraocular pressure elevation, and retinal detachment. However, when using that system, it is difficult to describe differences when the lesion is limited to the sclera. Another grading system described by Sen et al. [7] uses photograph-based methods. Grades are determined by the extent of redness and dilation of scleral vessels. This is a practical system for evaluating the severity of anterior scleritis. However, when calculating mild and modest grades $(0.5,1$, and 2$)$, the scores may vary subjectively according to individual graders. To clarify these problems, we have established a new grading system that can assess the severity of scleritis based on common clinical signs, such as the area of inflammation in sclera and ocular pain. This system is simple, rapid, and convenient. In the present study, this grading system was employed to examine its advantages in comparing clinical outcomes.

Scleritis often presents with uveitis and retinitis, and it can affect the extraocular muscles. In this study, the first case involved complications with iritis and the second showed complications of lacrimal gland swelling without any symptoms. In both cases, inflammation was controlled successfully through treatment.

Although scleritis is not a rare ocular disease, its response to therapy can vary among individual cases, making it difficult to establish a standard protocol of treatment. Topical corticosteroids are usually the first choice of treatment for noninfectious scleritis, but the success rate is reported to be under 50\% [8]. Recently, subconjunctival triamcinolone injection treatment has been lauded as an effective means to combat scleritis that does not respond to topical corticosteroids [9]. This treatment was applied to our first case, but remission was minimal.

For cases that are resistant to topical corticosteroids, systemic corticosteroids are widely considered to be the treatment of choice. Oral prednisolone is commonly administered, and intravenous pulse methylprednisolone is occasionally applied to persistent cases [10, 11]. In our study, both cases were treated with oral prednisolone with unsatisfactory results. In addition, pulse therapy was performed twice in the first patient, but results were insuffi- 
Aoki et al.: Systemic Cyclosporine Therapy for Scleritis: A Proposal of a Novel System to Assess the Activity of Scleritis

cient. The long-term application of corticosteroids increases the risk of side effects, including elevated blood glucose. Neither of our cases showed any systemic side effects, but they had an elevation in intraocular pressure. This made it necessary to apply an alternative therapy.

Corticosteroid treatment is the first line of scleritis therapy; however, other immunosuppressive agents are applied additionally when conditions are poorly controlled with corticosteroids alone or when side effects from the corticosteroids make an alternative treatment necessary. Recently, cyclosporine has been recognized as an effective therapeutic agent for several ocular pathologies such as uveitis and scleritis; additionally, it helps prevent rejection after corneal transplantation. It is reported that $2-3.5 \mathrm{mg} / \mathrm{kg} /$ day of cyclosporine is effective and has a low risk of side effects. The ideal trough value ranges from 50 to $150 \mathrm{ng} / \mathrm{ml}$ [5]. Although the two patients in our study had similar body weights (approximately $50 \mathrm{~kg}$ ) and initially were administered similar amounts of cyclosporine, there was a great difference between the two cases in the drug concentration of the serum. The trough value in the first case did not exceed $50 \mathrm{ng} / \mathrm{ml}$ until cyclosporine administration was increased to $200 \mathrm{mg} /$ day. Conversely, the trough value in the second case was greater than $100 \mathrm{ng} / \mathrm{ml}$ even when cyclosporine administration was reduced to $100 \mathrm{mg} /$ day. This discrepancy may depend on the metabolic differences in each patient. The outcomes of our cases suggest that a trough value of approximately $100 \mathrm{ng} / \mathrm{ml}$ is efficient to control scleritis. The dosage should be monitored to reduce the overall exposure to the drug and still achieve disease remission.

The present findings suggest the importance of exploring the trough value of cyclosporine in order to control scleritis without side effects. Further investigations with increased numbers of case studies and statistical analysis may result in the establishment of a standard protocol to treat scleritis.

\section{Statement of Ethics}

The present study protocol was approved by the Ethics Committee of the Sapporo Medical University School of Medicine and conducted in accordance with the Declaration of Helsinki. After a full explanation of the purpose and protocol for this study was provided to the patients, informed consent was obtained.

\section{Disclosure Statement}

The authors declare that they have no conflicts of interest.

\section{References}

1 Lin P, Bhullar SS, Tessler HH, Goldstein DA: Immunologic markers as potential predictors of systemic autoimmune disease in patients with idiopathic scleritis. Am J Ophthalmol 2008;145:463-471.

2 Watson PG, Hayreh SS: Scleritis and episcleritis. Br J Ophthalmol 1976;60:163-191.

-3 Watson PG: Doyne Memorial Lecture, 1982. The nature and the treatment of scleral inflammation. Trans Ophthalmol Soc UK 1982;102:257-281.

4 Meyer PA, Watson PG, Franks W, Dubord P: 'Pulsed' immunosuppressive therapy in the treatment of immunologically induced corneal and scleral disease. Eye (Lond) 1987;1:487-495.

-5 Kacmaz RO, Kempen JH, Newcomb C, et al: Cyclosporine for ocular inflammatory diseases. Ophthalmology 2010;117:576-584.

6 Wakefield D, McCluskey P: Cyclosporin therapy for severe scleritis. Br J Ophthalmol 1989;73:743-746. 
Aoki et al.: Systemic Cyclosporine Therapy for Scleritis: A Proposal of a Novel System to Assess the Activity of Scleritis

7 Sen HN, Sangave AA, Goldstein DA, et al: A standardized grading system for scleritis. Ophthalmology 2011;118:768-771.

8 McMullen M, Kovarik G, Hodge WG: Use of topical steroid therapy in the management of nonnecrotizing anterior scleritis. Can J Ophthalmol 1999;34:217-221.

-9 Sohn EH, Wang R, Read R, et al: Long-term, multicenter evaluation of subconjunctival injection of triamcinolone for non-necrotizing, noninfectious anterior scleritis. Ophthalmology 2011;118:1932-1937.

10 Sainz de la Maza M, Jabbur NS, Foster CS: An analysis of therapeutic decision for scleritis. Ophthalmology 1993;100:1372-1376.

-11 McCluskey P, Wakefield D: Intravenous pulse methylprednisolone in scleritis. Arch Ophthalmol 1987;105:793-797.

Table 1. Scoring system for scleritis

\begin{tabular}{llll}
\hline Area of congestion & Ocular pain & \\
\hline 0 & None & 0 (none) & \\
1 & One quadrant & 1 (mild) & Analgesic unnecessary \\
2 & Two quadrants & 2 (moderate) & Analgesic necessary \\
3 & Three quadrants & 3 (severe) & Analgesic insufficient \\
4 & Whole & & \\
\hline
\end{tabular}


Aoki et al:: Systemic Cyclosporine Therapy for Scleritis: A Proposal of a Novel System to Assess the Activity of Scleritis
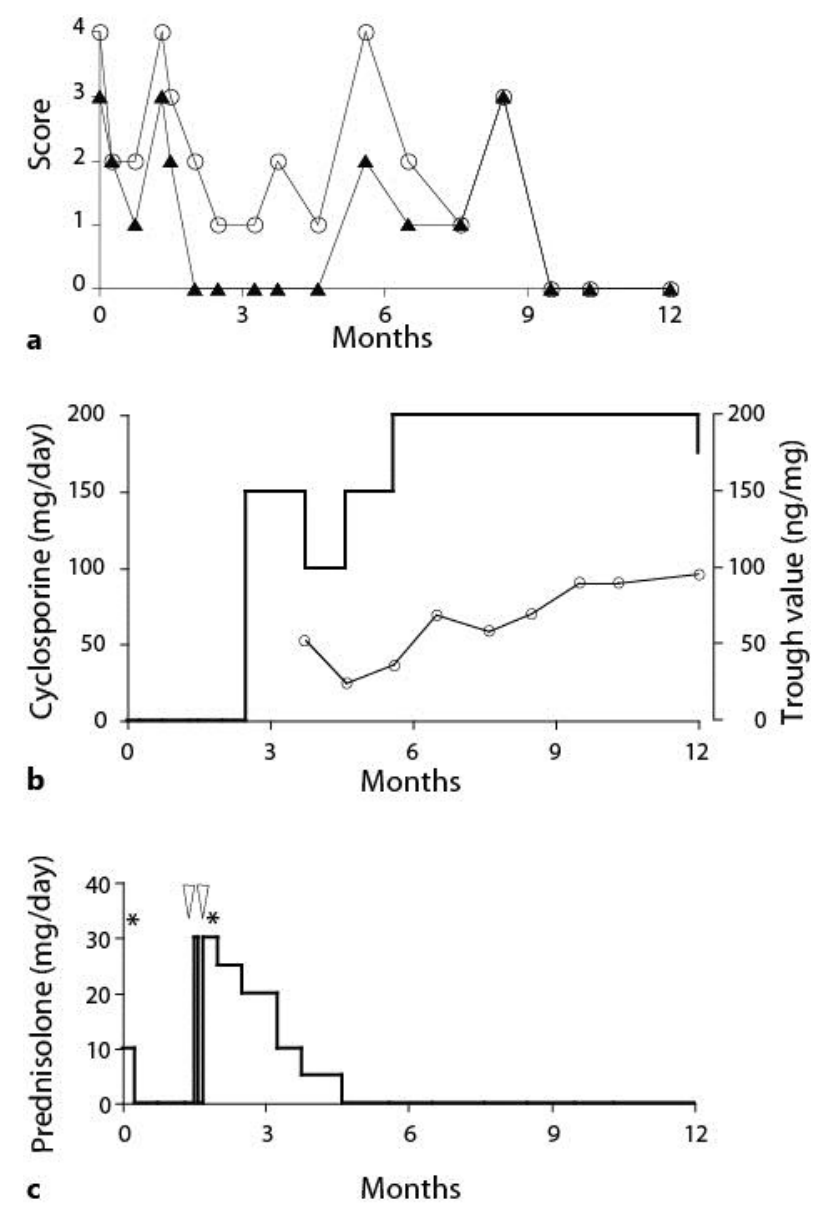

Fig. 1. The clinical course of patient 1. a The extent of anterior scleritis in the right eye. The vertical axis shows the scleritis score. The congestion scores are displayed as triangles, and the ocular pain scores are displayed as circles. $\mathbf{b}$ Cyclosporine application and serum concentration. The columns indicate the dose of oral cyclosporine. Dose volume is indicated by the left vertical axis. Circles indicate the dosage of serum cyclosporine concentration as trough value. The dosage is indicated by the right vertical axis. c Administration of corticosteroids. The columns indicate the dose of oral prednisolone. Dose volume is indicated by the left vertical axis. The triangles indicate the application of corticosteroid pulse therapy, and asterisks indicate the subconjunctival triamcinolone injection. The horizontal axis displays the duration of observation. 
Case Reports in

Ophthalmology

\begin{tabular}{l|l}
\hline \multicolumn{2}{l}{ Case Rep Ophthalmol 2015;6:149-157 } \\
\hline DOI: 10.1159/000430490 & $\begin{array}{l}\text { ○ 2015 S. Karger AG, Basel } \\
\text { www.karger.com/cop }\end{array}$ \\
\hline
\end{tabular}

Aoki et al.: Systemic Cyclosporine Therapy for Scleritis: A Proposal of a Novel System to Assess the Activity of Scleritis

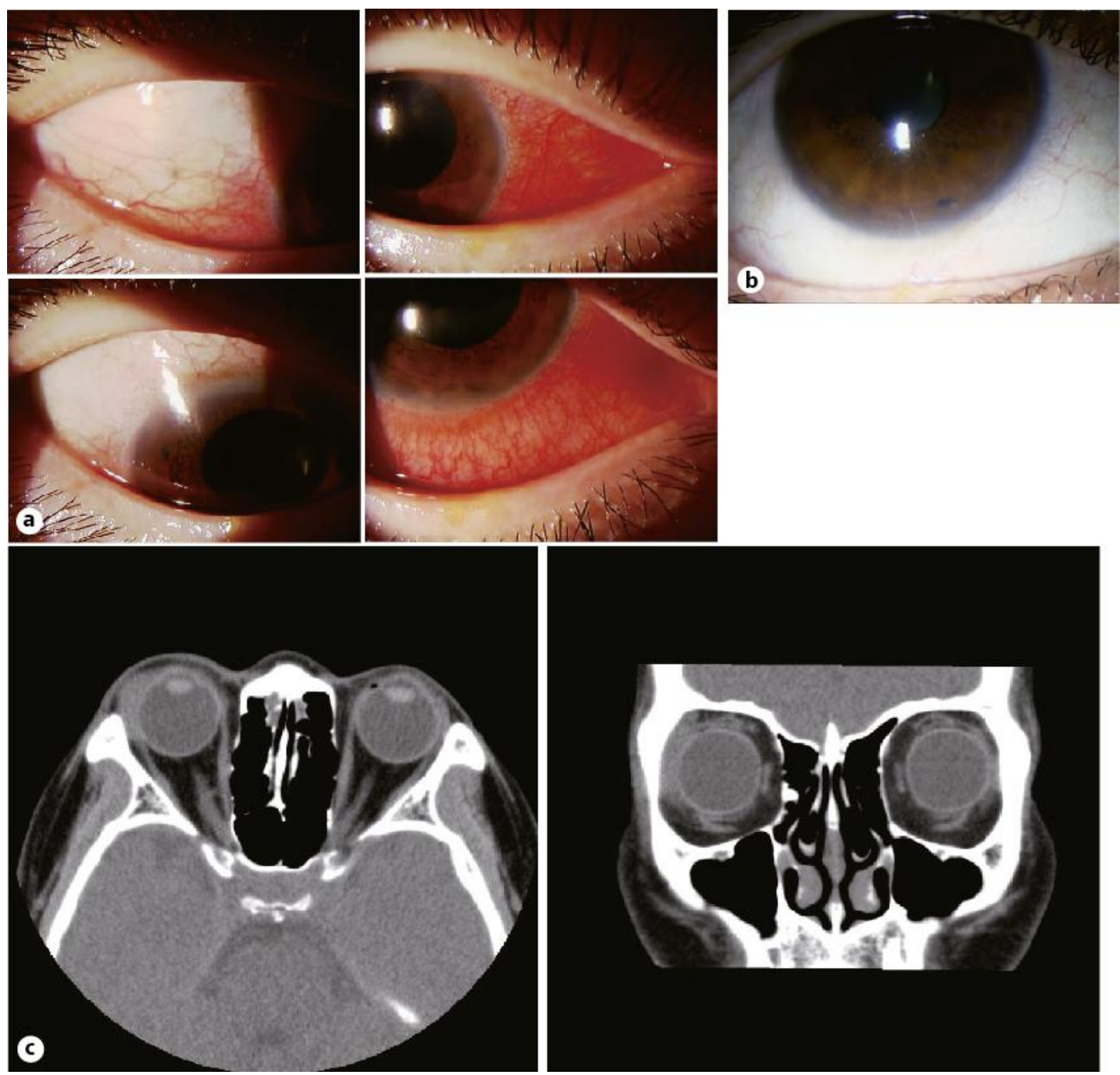

Fig. 2. a Slit-lamp photographs of case 1 at month 8 with congestion in three quadrants of the anterior sclera, which is defined as a score of 3. b Slit-lamp photograph of case 1 in month 10 without congestion in the anterior sclera, which is defined as a score of 0 . c Computed tomographic scanning image of the head of case 2; the horizontal section (left panel) and coronal section (right panel) showed right lacrimal gland enlargement. 
Aoki et al.: Systemic Cyclosporine Therapy for Scleritis: A Proposal of a Novel System to Assess the Activity of Scleritis
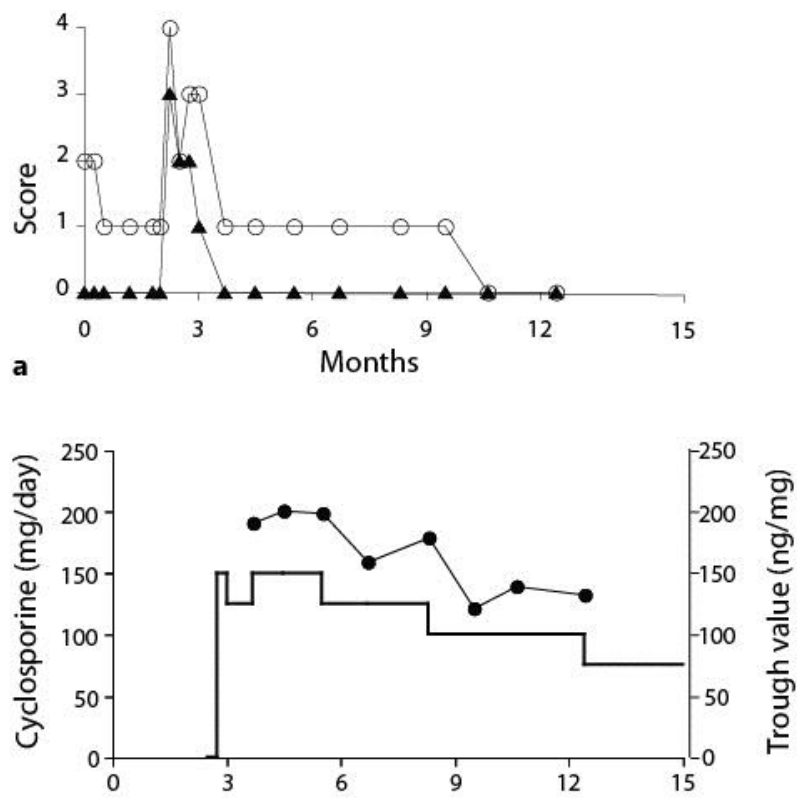

b

Months

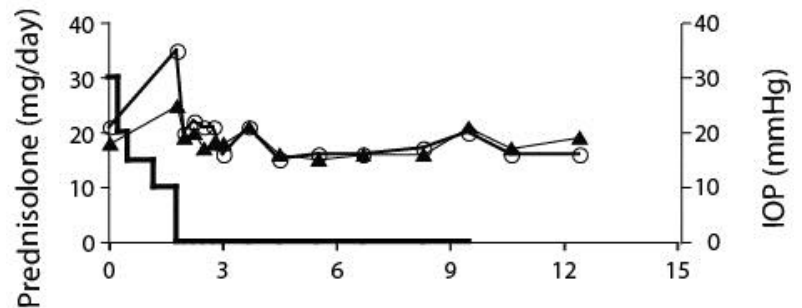

c Months

Fig. 3. The clinical course of patient 2. a The extent of anterior scleritis in the right eye. b Cyclosporine application and serum concentration. $\mathbf{c}$ The administration of corticosteroids is indicated in columns, and the intraocular pressure is indicated in circles (right eye) or in triangles (left eye). The notations are the same as those in figure 1. 